University of Nebraska - Lincoln

DigitalCommons@University of Nebraska - Lincoln

April 1996

\title{
Effects of coherent coupling of autoionizing states on multiphoton ionization
}

\author{
N. E. Karapanagioti \\ Foundation for Research and Technology-Hellas, Institute of Electronic Structure and Laser, 71110 \\ Heraklion, Crete, Greece \\ D. Charalambidis \\ Foundation for Research and Technology-Hellas, Institute of Electronic Structure and Laser, 71110 \\ Heraklion, Crete, Greece \\ Cornelis J. Uiterwaal \\ University of Nebraska - Lincoln, cuiterwaal2@unl.edu \\ C. Fotakis \\ Foundation for Research and Technology-Hellas, Institute of Electronic Structure and Laser, 71110 \\ Heraklion, Crete, Greece \\ H. Bachau \\ Centre de Physique Theorique MB, CNRS URA 1537, Laboratoire de Collisions Atomiques, Université de \\ Bordeaux I, 351 Cours de Libération, 33405 Talence, France \\ See next page for additional authors \\ Follow this and additional works at: https://digitalcommons.unl.edu/physicsuiterwaal \\ Part of the Physics Commons
}

Karapanagioti, N. E.; Charalambidis, D.; Uiterwaal, Cornelis J.; Fotakis, C.; Bachau, H.; Sanchez, I.; and Cormier, E., "Effects of coherent coupling of autoionizing states on multiphoton ionization" (1996). C.J.G.J. Uiterwaal Publications. 7.

https://digitalcommons.unl.edu/physicsuiterwaal/7

This Article is brought to you for free and open access by the Research Papers in Physics and Astronomy at DigitalCommons@University of Nebraska - Lincoln. It has been accepted for inclusion in C.J.G.J. Uiterwaal Publications by an authorized administrator of DigitalCommons@University of Nebraska - Lincoln. 


\section{Authors}

N. E. Karapanagioti, D. Charalambidis, Cornelis J. Uiterwaal, C. Fotakis, H. Bachau, I. Sanchez, and E. Cormier

This article is available at DigitalCommons@University of Nebraska - Lincoln: https://digitalcommons.unl.edu/ physicsuiterwaal/7 
Phys. Rev. A 53, 2587 - 2597 (1996)

[Issue 4 - April 1996]

\title{
Effects of coherent coupling of autoionizing states on multiphoton ionization
}

\author{
N. E. Karapanagioti, D. Charalambidis, C. J . G. J . Uiterwaal, and C. \\ Fotakis \\ Foundation for Research and Technology-Hellas, Institute of Electronic \\ Structure and Laser, P.O. Box 1527, 71110 Heraklion, Crete, Greece \\ H. Bachau, I. Sánchez, and E. Cormier \\ Centre de Physique Theorique MB, CNRS URA 1537, Laboratoire de \\ Collisions Atomiques, Université de Bordeaux I, 351 Cours de Libération, \\ 33405 Talence, France
}

Received 17J uly 1995

We present the experimental and theoretical investigation of the effects of the strong electromagnetic coupling of two autoionizing states (AIS's) on the photoionization properties of an atomic system. These effects are observed by probing the ladder system from the ground state of the atom through a weak two-photon transition to the region of the lower AIS. The experimental observation of the modification of the total ionization and partial photoelectron spectra at different wavelengths of the coupling and probe lasers are accurately reproduced by a theoretical analysis which uses a density-matrix approach. Both theory and experiment indicate a strong inhibition of the ionization of the system in the case when both lasers are resonant with the two atomic transitions, while displaying a rapidly varying asymmetry induced as the detuning between the two states and the fields increases. In the extreme case of a large detuning, two separate structures appear as distinct total ionization and photoelectron peaks. The above findings combined with the full theoretical treatment indicate that the phenomena observed are an experimental demonstration of ac Stark splitting between two AIS's.

(C)1996 The American Physical Society. Used by permission.

URL: http:// link.aps.org/ abstract/ PRA/v53/p2587

DOI: 10.1103/ PhysRevA.53.2587

PACS: 32.80.Rm, 32.80.Qk, 32.80.Dz 


\title{
Effects of coherent coupling of autoionizing states on multiphoton ionization
}

\author{
N. E. Karapanagioti, D. Charalambidis, ${ }^{*}$ C. J. G. J. Uiterwaal, and C. Fotakis* \\ Foundation for Research and Technology-Hellas, Institute of Electronic Structure and Laser, P.O. Box 1527, \\ 71110 Heraklion, Crete, Greece \\ H. Bachau, I. Sánchez, ${ }^{\dagger}$ and E. Cormier \\ Centre de Physique Theorique MB, CNRS URA 1537, Laboratoire de Collisions Atomiques, Université de Bordeaux I, \\ 351 Cours de Libération, 33405 Talence, France
}

(Received 17 July 1995)

\begin{abstract}
We present the experimental and theoretical investigation of the effects of the strong electromagnetic coupling of two autoionizing states (AIS's) on the photoionization properties of an atomic system. These effects are observed by probing the ladder system from the ground state of the atom through a weak two-photon transition to the region of the lower AIS. The experimental observation of the modification of the total ionization and partial photoelectron spectra at different wavelengths of the coupling and probe lasers are accurately reproduced by a theoretical analysis which uses a density-matrix approach. Both theory and experiment indicate a strong inhibition of the ionization of the system in the case when both lasers are resonant with the two atomic transitions, while displaying a rapidly varying asymmetry induced as the detuning between the two states and the fields increases. In the extreme case of a large detuning, two separate structures appear as distinct total ionization and photoelectron peaks. The above findings combined with the full theoretical treatment indicate that the phenomena observed are an experimental demonstration of ac Stark splitting between two AIS's.
\end{abstract}

PACS number(s): $32.80 . \mathrm{Qk}, 32.80 . \mathrm{Rm}, 32.80 . \mathrm{Dz}$

\section{INTRODUCTION}

Coherent interactions of an atomic system with two electromagnetic fields in the so-called $\Lambda, \mathrm{V}$, and ladder coupling schemes have been the subject of a vast number of theoretical studies for many years now. In the last ten years, the subject has received widespread attention because of several experimental observations of related phenomena involving bound states but also smooth or structured continua. Laserinduced continuum structure (LICS) [1] in smooth and structured continua, electromagnetically induced transparency (EIT) [2], coherent population trapping (CPT) [3], adiabatic population transfer (APT) [4], and resonance stabilization [5] are representative examples of the recently investigated and experimentally demonstrated effects. This paper presents the experimental results and theoretical calculations relating to the drastic modification of the ionization dynamics of an atomic system in the vicinity of an autoionizing state (AIS). This modification is achieved through the strong electromagnetic coupling of the state to a higher-lying autoionizing resonance by means of visible laser radiation. The modified autoionizing structure is probed by a weaker laser from the ground state of the atom. As shown in a recent Letter [6] (referred to as paper I), the resonant coupling of the two AIS's results in a drastic reduction of the ionization when the coupling laser intensity reaches the order of $10^{10} \mathrm{~W} / \mathrm{cm}^{2}$. This partial stabilization of autoionization is shown to be due to the coherent population inhibition of the faster-decaying

\footnotetext{
*Also at Physics Department, University of Crete, Greece.

†Present address: Departamento de Química C-9, Universidad Autónoma de Madrid, 28049-Madrid, Spain.
}

probed AIS. The present paper produces these observations in further detail and extends the results to cases when the modifying laser is near resonance, reporting on the shape of the autoionizinglike structure induced in the broad profile of the AIS, as well as its dramatically varying asymmetry as a function of the sign and magnitude of the detuning. Moreover, it studies the more general case of the coupling laser being detuned far from resonance, exploring the possibility of achieving laser-induced continuum structure through the embedding of the higher AIS to a lower-lying continuum, in an above-threshold ionization process. This study involves both ion and energy-selected photoelectron spectra, the latter adding complementary information on the decay mechanism that serves towards the precise understanding and interpretation of the observations. The scheme under investigation involves the $3 p^{2}\left({ }^{1} S^{e}\right)$ autoionizing state of $\mathrm{Mg}$, which is particularly broad $\left(\approx 300 \mathrm{~cm}^{-1}\right)$ due to its two highly correlated electrons. This state was strongly coupled by an intense electromagnetic field to the higher-lying and much narrower $3 p 3 d\left({ }^{1} P^{o}\right)$ AIS, as indicated in Fig. 1. The weaker laser

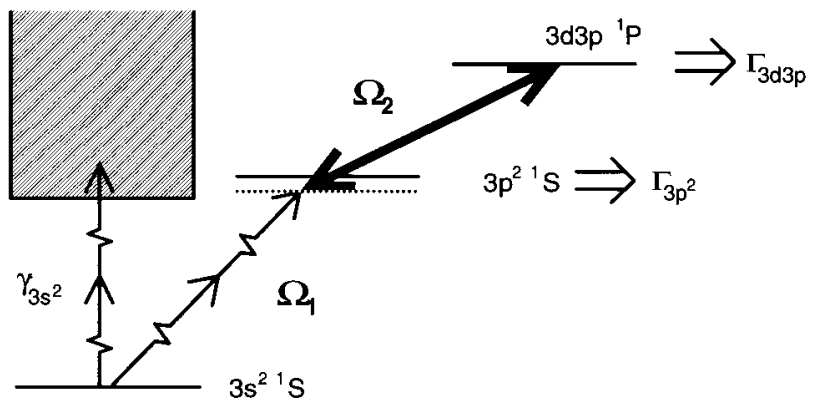

FIG. 1. Partial energy level diagram of magnesium indicating the laser couplings and the relevant atomic states. 


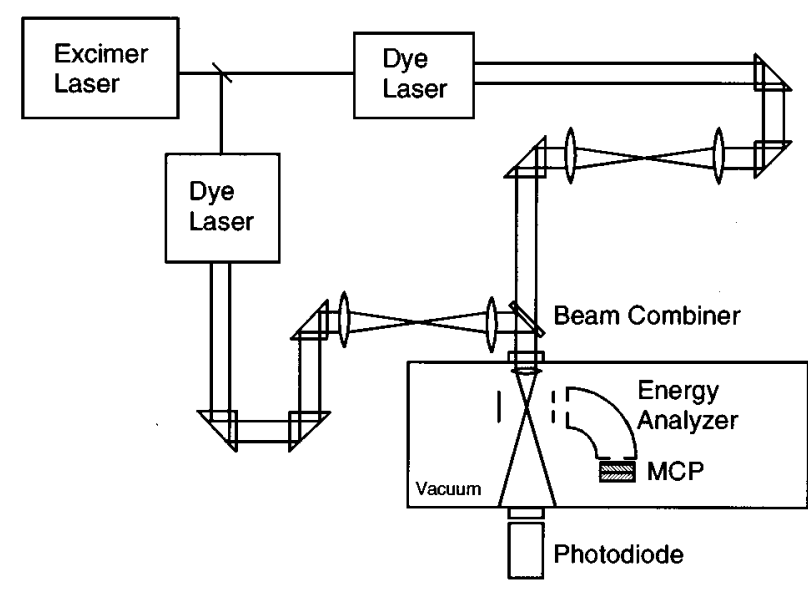

FIG. 2. Schematic diagram of the experimental layout.

probed the $3 p^{2}\left({ }^{1} S^{e}\right)$ profile from the ground state via twophoton excitation.

The present work, to our knowledge, is the first conclusive experiment which together with its theoretical counterpart demonstrates the effects of a strong electromagnetic coupling between two autoionizing states. Considering the significant theoretical interest in the subject during the past decade $[7,8]$, as well as the numerous possible applications with respect to short wavelength generation schemes (harmonic generation, amplification without inversion), this investigation can open up new paths for the exploration of coherent interactions into far regions of the continuum.

\section{EXPERIMENTAL DESCRIPTION}

In the experimental work described, the coupling and probing of the states was achieved by two excimer-pumped, tunable dye lasers (Lambda Physik LPD3000). The pulses produced had a duration of around $10 \mathrm{~ns}$ and a bandwidth of $5 \mathrm{GHz}$, and the laser wavelength was known with an accuracy of $0.1 \mathrm{~nm}$. The probe laser (laser 1) was frequency doubled by a $\beta-\mathrm{BaB}_{2} \mathrm{O}_{4}(\mathrm{BBO})$ and a potassium dihydrogen phosphate (KDP) crystal to produce the UV photons necessary for the two-photon transition to the $3 p^{2}$ magnesium state $\left(\lambda_{1} \approx 293 \mathrm{~nm}\right)$, while the coupling laser (laser 2$)$ was used in the visible $\left(\lambda_{2} \approx 566 \mathrm{~nm}\right)$. The beams were combined by a dichroic mirror and directed copropagatingly into the vacuum chamber, where they were focused by an $f=5 \mathrm{~cm}$ lens, as shown in Fig. 2. Both lasers were linearly polarized with parallel polarizations. To ensure that the two beams would focus at the same position, a telescope was introduced into each of the beam paths to slightly alter their divergence, and these were optimized for maximum beam overlap. The estimated intensities achieved in the interaction region were of the order of $10^{8}$ and $0.5 \times 10^{10} \mathrm{~W} / \mathrm{cm}^{2}$ for the probe and coupling lasers, respectively. However, the actual value of the intensity could be up to a factor of 4 off, due to the known uncertainties when measuring the beam spatial distribution around the focus. Moreover, despite the efforts to ensure the best beam overlap possible, the different beam sizes and divergences resulted in only partial overlap, so that part of the ionization from the probe laser was produced in a region where the coupling laser was not present.

The atomic beam was produced by heating the magne- sium in an oven at $410{ }^{\circ} \mathrm{C}$, whence it came out through a 1 -mm aperture into the chamber $\left(P \approx 10^{-6} \mathrm{mbar}\right)$. The resulting number density was estimated to be approximately $10^{9}$ atoms $/ \mathrm{cm}^{3}$, and the beam was directed at right angles to the lasers and to the detection axis. The decay of the autoionizing states populated by the lasers led to the production of ions and electrons, which were detected by a short time-offlight (TOF) mass spectrometer and an electrostatic energy analyzer, respectively, at different stages of the experiment. The TOF setup basically consisted of a repeller plate biased at $300 \mathrm{~V}$ which directed the ions towards a 5-mm-wide entrance slit and thereon to a double microchannel plate detector. The slit was used in order to ensure that the detection volume was confined around the laser focus where the coupling intensity was strong enough to induce the phenomena under investigation, thus reducing the signal contribution from the background ionization far from focus. This experimental configuration produced spectra of the total ionization versus different sets of probe and coupling laser wavelengths. Although in the experiment the TOF was used simply as an ionization detector, it was employed initially as a mass spectrometer to ensure that there were minimal impurities in our spectrum.

The employment of the energy analyzer enabled the recording of spectra of the photoelectrons produced by the decay of the $3 p^{2}$ and the $3 p 3 d$ states separately. Since these electrons were ejected at widely different energies $(0.8$ and 3 $\mathrm{eV}$, respectively), the instrument resolution $(\approx 0.4 \mathrm{eV})$ was sufficient to separate them and only record one of these at a time. The setup used has been described in detail elsewhere [9]. Briefly, after the photoelectrons were produced, they were repelled by a potential of $-10 \mathrm{~V}$ into a $120^{\circ}$ spherical sector electrostatic energy analyzer. According to the voltages applied on the entrance aperture and sector sides of the analyzer, electrons of different energies were transmitted onto the double microchannel plate detector at the end of the instrument. The analyzer was operated at the constant transmission mode. Moreover, additional tests cross-checking the instrumental efficiency versus existing results [10] proved that this remained effectively the same for $1-$ and $3-\mathrm{eV}$ photoelectrons, thus allowing a detailed and quantitative comparison between the two types of spectra. Both the TOF and energy analyzer signal were led, after amplification, to a boxcar integrator and then to a computer.

Of the two detection methods used, the analyzer had the obvious advantage of being able to distinguish between the different decay mechanisms that led to the production of the signal. However, the sensitivity of the TOF detector was much greater as it had a larger signal-to-noise ratio and enabled the observation of finer spectral details, thus making the two techniques employed complementary.

\section{EXPERIMENTAL RESULTS}

The experimental results obtained with the setup described above comprise excitation spectra of total ionization as well as energy-resolved photoelectrons. In each case, two types of data were obtained: (a) scans of the probe laser wavelength $\lambda_{1}$ at a fixed value of the coupling wavelength $\lambda_{2}$, and (b) scans of $\lambda_{2}$ with the probe laser $\lambda_{1}$ fixed. Figures 3(a) and 3(b) display one such pair of scans: Fig. 3(a) exhib- 

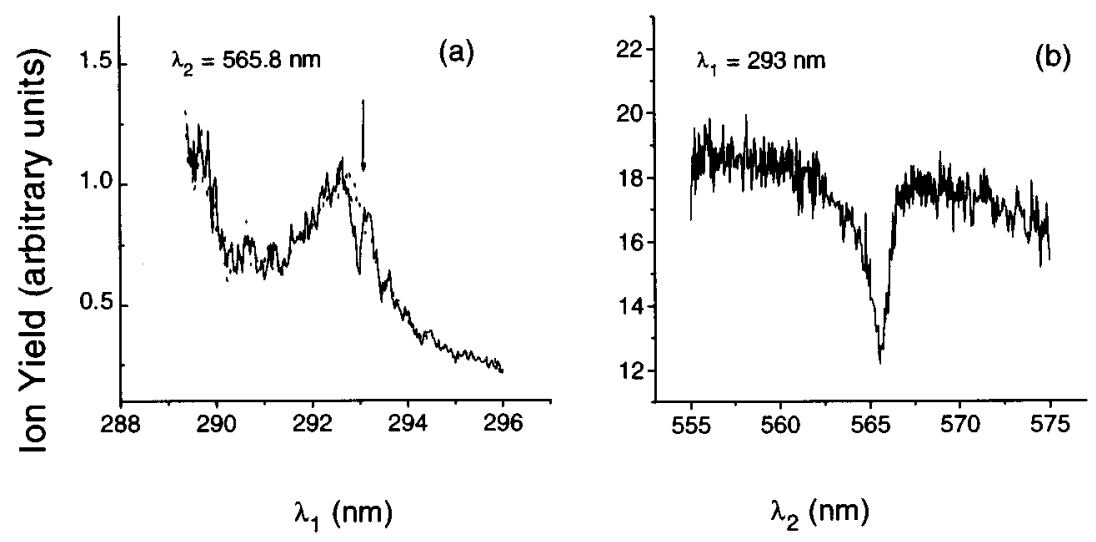

FIG. 3. Total ionization spectra: (a) scan of the probe laser through the $3 p^{2}$ resonance with the coupling laser fixed on the $3 p^{2}-3 p 3 d$ resonance. The dashed line indicates the spectrum of the $3 p^{2}$ state obtained without the coupling laser. The data have been normalized with laser intensity; (b) corresponding scan of the coupling laser with the probe laser fixed at the position of the induced structure [arrow on (a)]. Both scans indicate a drastic reduction of the ionization when the resonance condition is fulfilled.

its a window in ionization induced approximately at the center of the $3 p^{2}$ peak by its resonant coupling to the $3 p 3 d$ through $\lambda_{2}$. This spectrum has been normalized with the square of laser intensity for reasons of comparison with the calculated spectrum. Appropriate tests were made in order to ensure that the two-photon transition was not saturated. Figure 3(b) depicts the equivalent scan of $\lambda_{2}$, which demonstrates clearly the shape of the induced structure. For this correspondence to exist between the two data sets, in scan (b) $\lambda_{1}$ has to be fixed at the position at which the induced structure appears in scan (a). This ensures that the detuning is the same for both cases (a) and (b), as required by the resonance condition for the induced structure, i.e., that $\delta_{1}=-\delta_{2} \quad\left[\delta_{1}=2 \hbar \omega_{1}-\left(E_{3 p^{2}}-E_{3 s^{2}}\right) \quad\right.$ and $\quad \delta_{2}=\hbar \omega_{2}$ $-\left(E_{3 p 3 d}-E_{3 p^{2}}\right)$, see theory, Sec. V]. In this set of scans, the dip observed displays a slight asymmetry which may be attributed to the fact that $\lambda_{1}$ is not fixed exactly at the center of the $3 p^{2}$ resonance. A particularly interesting aspect of the phenomenon observed is the variation of the shape of the induced structure when $2 \hbar \omega_{1}$ is not at the maximum of the $3 p^{2}$ resonance. Such cases are shown in Fig. 4(a), which displays a number of total ionization scans of the coupling laser with $\lambda_{1}$ fixed at various points of the two-photon resonance to the $3 p^{2}$ state, as given by the detuning $\delta_{1}$. The corresponding scans of $\lambda_{1}$ by keeping $\lambda_{2}$ fixed at different values also show induced autoionizinglike structures at positions of the $3 p^{2}$ profile that satisfy the energy balance. As can be seen in Fig. 4, the asymmetry of the structure induced by the coupling varies drastically as a function of the detuning from the center of the resonance. It is also apparent that an asymmetry reversal occurs upon change of the detuning sign. Due to its asymmetry, we can describe the induced structure as a Fano profile [11] and assign to it a $q$ parameter, although this parameter is not formally defined in any way. In these spectra, $q$ appears to take a value starting from 0 , as in the case of a window resonance, when $\delta_{1}$ is $\approx 0$. It then takes on a small value at the side of the resonance $\left(\delta_{1}=-202 \mathrm{~cm}^{-1}\right)$ and at a large detuning $\left(\delta_{1}=-463\right.$ $\left.\mathrm{cm}^{-1}\right), q$ also becomes large and the structure becomes more symmetric. What is also evident is that at positive values of the detuning, the asymmetry is also reversed, giving near- equal but opposite values of $q$ to the induced structure. The differences between the corresponding scans of negative and positive detuning (e.g., $\delta_{1}=-463$ and $469 \mathrm{~cm}^{-1}$ ) may be due to the fact that the profile of the $3 p^{2}$ state is not totally symmetric due to near-resonantly enhanced multiphoton ionization through the $3 s 3 p\left({ }^{1} P^{o}\right)$ bound state which occurs on the short wavelength part of the scan. Note that going over the $3 p^{2}$ resonance, the $3 s^{2}-3 p 3 d$ three-photon coupling rapidly changes amplitude and sign. This accounts for the observed dip, and the asymmetry and asymmetry reversal of the induced structure. It is worth mentioning that this is nothing else than an equivalent representation of ac Stark splitting in the dressed atom picture. In the latter, window resonances and asymmetry reversals can be interpreted by means of fully or partially destructive interference from the two Autler-Townes components depending on the value and sign of the detuning of the coupling that causes the ac Stark splitting.

As the detuning increases beyond the width of the $3 p^{2}$ state, the structure observed becomes an almost symmetric and separate ionization peak on the side of the $3 p^{2}$ resonance (see Fig. 5). The precise nature of this feature, however, cannot be determined with only these data at hand, since the origin of the ionization mechanism is not known. The peak may correspond to increased ionization rate near the $3 p^{2}$ state, or it might be due to ionization from the $3 p 3 d$ state, which is now three-photon resonant $\left(2 \hbar \omega_{1}+\hbar \omega_{2}\right)$. The latter has been suggested by Gea-Banacloche et al. [12] as the interpretation of their results in a similar ladder scheme involving states below the threshold.

To clarify the above question, the energy-resolved photoelectron spectra were obtained. As mentioned in the preceding section, the analyzer allowed us to record separate signals from the energy region of each of the two AIS's, and thus to perform scans relating to the decay of each of these states independently. Upon conducting this stage of the experiment, the main realization was that the spectra of the photoelectrons originating from decay in the region of the $3 p^{2}$ state were practically identical to the total ionization spectra. They displayed the effects already outlined above in relation to total ionization (e.g., signal reduction in the center 
(a)

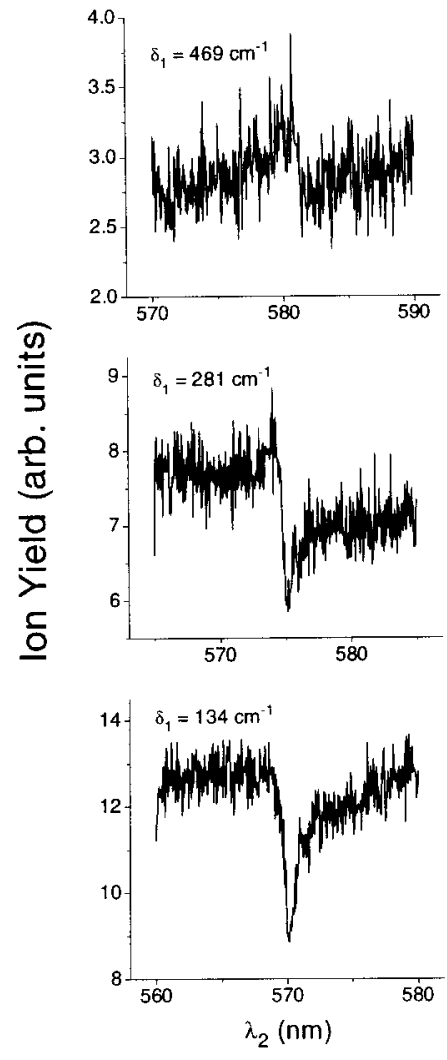

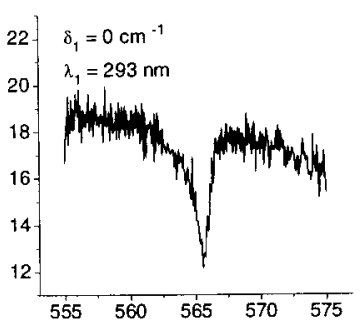
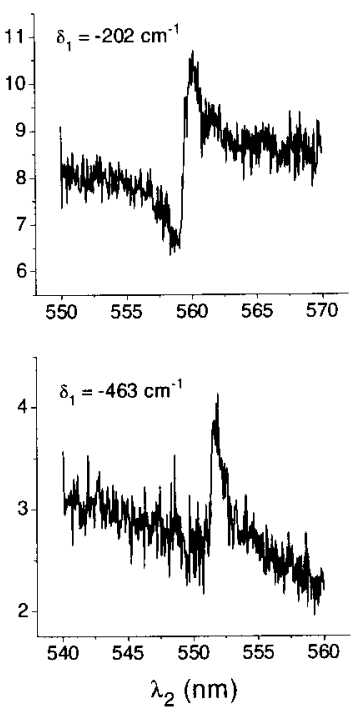

(b)

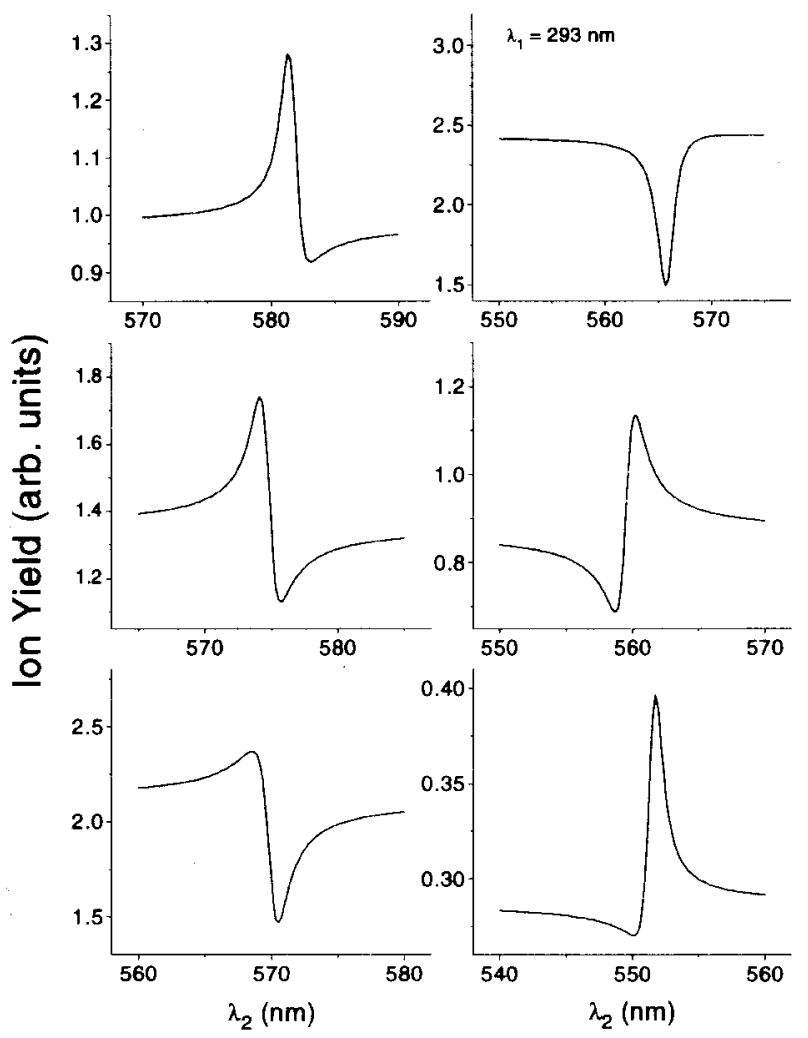

FIG. 4. (a) Total ionization spectra of scans of the coupling laser versus fixed values of the probe laser wavelength. As $\lambda_{1}$ is fixed at different positions of the two-photon resonance to the $3 p^{2}$ state, the induced structure changes asymmetry as we go from negative to positive detuning. The position of zero detuning has been arbitrarily set at $\lambda_{1}=293 \mathrm{~nm}$. (b) Corresponding theoretical spectra, at a probe laser intensity of $10^{8} \mathrm{~W} / \mathrm{cm}^{2}$ and a coupling laser intensity of $0.25 \times 10^{10} \mathrm{~W} / \mathrm{cm}^{2}$.

of the state of $30 \%$ ), and more specifically, as shown in Fig. 6 , at large laser detunings a separate photoelectron peak appeared in the region of the $3 p^{2}$ AIS. Due to the increased noise, the peaks induced at various coupling laser wavelengths are shown in magnification, where it is clear that they also demonstrate asymmetry changes, tending towards a more symmetric profile at larger detunings.

In addition to these observations, it also emerged that the signal obtained from the region of the $3 p 3 d$ state was extremely weak, less than $10 \%$ of the $3 p^{2}$ one. Very faint spectra of this vicinity were obtained through pulse counting techniques, indicating the appearance of the resonance as the coupling laser was scanned through it (see Fig. 7). This made it clear that almost all of the ionization recorded originated from decay of or around the $3 p^{2}$ state, with only a minimal contribution from the $3 p 3 d$.

\section{ATOMIC STRUCTURE CALCULATIONS}

In this section, we calculate the atomic parameters needed for the full dynamical treatment of the problem which is given in Sec. V.

\section{A. Theoretical approach, the Feshbach formalism}

We have evaluated the relevant parameters associated to the atomic levels involved in the process we are considering here. As in previous studies of two-valence electron systems (see, for example [13-16],) we have applied a frozen-core approximation, with a polarization potential. The core $\mathrm{Mg}^{2+}\left(1 s^{2} 2 s^{2} 2 p^{6}\right)$ is represented by the self-consistentfield wave function, so that the Hamiltonian for the valence shell is written

$$
\mathscr{H}_{v}=Q_{v}\left[h_{v}\left(\vec{r}_{1}\right)+h_{v}\left(\vec{r}_{2}\right)+\frac{1}{r_{12}}+V_{\mathrm{pol}}\left(\vec{r}_{1}, \vec{r}_{2}\right)\right] Q_{v},
$$

where $h_{v}\left(\vec{r}_{i}\right)$ is a one-electron Hamiltonian including the kinetic energy of valence electron $i$ as well as its interaction with the nucleus and core electrons (Coulomb and exchange terms), and $Q_{v}$ is a projector that ensures orthogonality to the Hartree-Fock core. Notice that this projector is necessary due to the introduction of $V_{\mathrm{pol}}\left(\vec{r}_{1}, \vec{r}_{2}\right)$, which is the polarization potential [16]:

$$
V_{\mathrm{pol}}\left(\vec{r}_{1}, \vec{r}_{2}\right)=V_{1}\left(\vec{r}_{1}\right)+V_{1}\left(\vec{r}_{2}\right)+V_{\text {diel }}\left(\vec{r}_{1}, \vec{r}_{2}\right),
$$

with

$$
V_{1}\left(\vec{r}_{i}\right)=-\alpha \sum_{l, m}\left|Y_{l}^{m}\right\rangle\left[W_{6}\left(\frac{r_{i}}{\beta_{l}}\right) \frac{1}{2 r_{i}^{4}}\right]\left\langle Y_{l}^{m}\right|,
$$




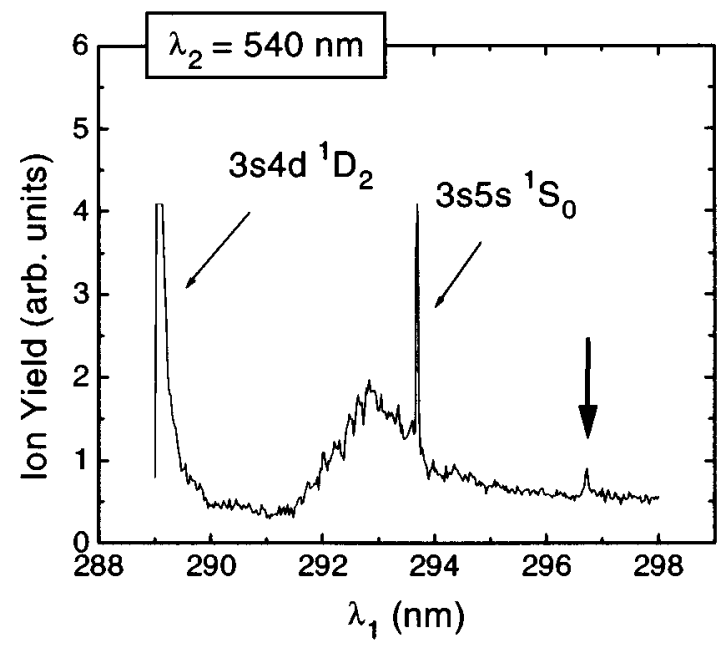

FIG. 5. Total ionization spectrum of the $3 p^{2}$ state with the coupling laser fixed far from the $3 p^{2}-3 p 3 d$ resonance. The induced structure appears on the right of the state as indicated by the thick arrow. The two narrow peaks are due to resonantly enhanced multiphoton ionization through accidental two-photon resonances $\left(\hbar \omega_{1}+\hbar \omega_{2}\right)$ to the states inscribed.

$$
\begin{gathered}
V_{\text {diel }}\left(\vec{r}_{1}, \vec{r}_{2}\right)=-\alpha \frac{\vec{r}_{1} \cdot \vec{r}_{2}}{r_{1}^{3} r_{2}^{3}} \sqrt{W_{6}\left(\frac{r_{1}}{B_{1}}\right) W_{6}\left(\frac{r_{2}}{B_{2}}\right)}, \\
W_{6}(x)=1-\exp \left(-x^{6}\right) .
\end{gathered}
$$

In the above equations, $Y_{l}^{m}$ is a spherical harmonic, and $\alpha$ the core dipole polarizability. Each cutoff parameter $\beta_{l}$ is adjusted in order to reproduce the experimental energy of ionization threshold $\mathrm{Mg}^{+}(n l)$, with $n=\max \{3, l+1\}$; on the other hand, when the matrix element of $V_{\text {diel }}\left(\vec{r}_{1}, \vec{r}_{2}\right)$ between two configurations $\left(n_{1} l_{1} n_{2} l_{2}\right)$ and $\left(n_{1}^{\prime} l_{1}^{\prime} n_{2}^{\prime} l_{2}^{\prime}\right)$ is calculated, $B_{i}$ is taken as the arithmetic mean of $\beta_{l_{i}}$ and $\beta_{l_{i}^{\prime}}$. The importance of the dielectronic term of the polarization potential, $V_{\text {diel }}\left(\vec{r}_{1}, \vec{r}_{2}\right)$, has been discussed in earlier works ([17], and references therein). We will just note that, if it is omitted, energies lower than the experimental ones can be obtained; consequently, the two-electron model would not be satisfactory.

The resonance parameters that appear in the densitymatrix equations have been calculated within the Feshbach formalism [18]. We now summarize the main equations. Our interest is focused on the energy range going from the first ionization threshold, $\mathrm{Mg}^{+}(3 s)$, to the second one, $\mathrm{Mg}^{+}(3 p)$. In this region, there is only one open channel per total orbital angular momentum, denoted as $3 \mathrm{skl}$, and ionization of the $\mathrm{Mg}$ atom leads to $\mathrm{Mg}^{+}$in its ground state. Therefore we define the Feshbach projector $P$ as (see [19])

$$
P=P_{1}+P_{2}-P_{1} P_{2},
$$

with

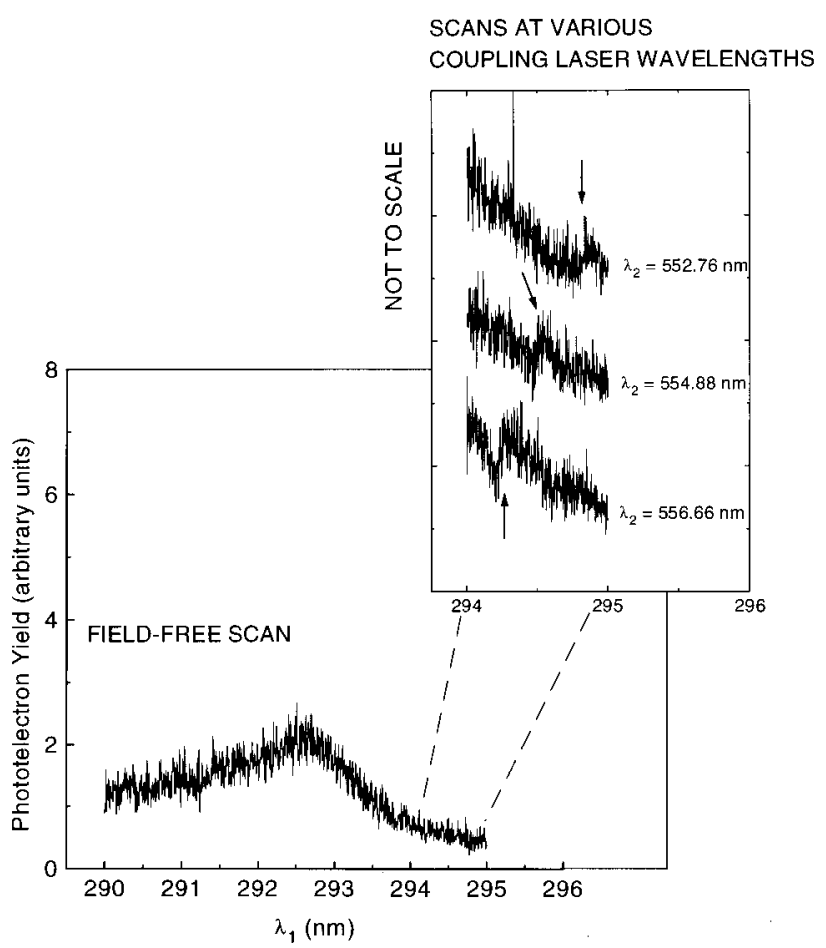

FIG. 6. Energy-selected photoelectron spectra around the $3 p^{2}$ AIS, relating to electron energies of $\approx 0.8 \mathrm{eV}$. The data were obtained by scanning $\lambda_{1}$ while fixing $\lambda_{2}$ at three values quite far detuned from the $3 p^{2}-3 p 3 d$ resonance. The top graphs display offset magnified sections of these scans with structure induced at different positions near the $3 p^{2}$ resonance profile (bottom graph).

$$
P_{i}=|3 s(i)\rangle\langle 3 s(i)|
$$

where $i$ stands for electron 1 or 2 , and $Q$ as the complement of $P$ :

$$
Q=1-P
$$

Then, Feshbach resonances embedded in continua converging to the second ionization threshold have a bound character represented by the eigenfunctions of $Q \mathscr{H}_{v} Q$ :

$$
\left(Q \mathscr{H}_{v} Q-\mathscr{E}_{n}\right) \phi_{n}^{L}=0
$$

$\phi_{n}^{L}$ defines the discrete part of the AIS, the autoionization width of state $\phi_{r}^{L}$ is given by

$$
\Gamma_{r}=2 \pi\left|\left\langle\phi_{r}^{L}\left|Q \mathscr{H} \mathscr{b}_{v} P\right| P \psi_{E}^{L, 0-}\right\rangle\right|^{2}
$$

where $\mathscr{E}_{r} \simeq E$, and $P \psi_{E}^{L, 0-}$ is a nonresonant continuum, solution of

$$
\begin{aligned}
\left(\mathscr{H}_{v}^{\prime}-E\right) P \psi_{E}^{L, 0-} & \\
\equiv & {\left[P \mathscr{H}_{v} P+\oint_{n \neq r} \frac{P \mathscr{H}_{v} Q\left|\phi_{n}^{L}\right\rangle\left\langle\phi_{n}^{L}\right| Q \mathscr{H}_{v} P}{\left(E-\mathscr{E}_{n}\right)}-E\right] } \\
& \times P \psi_{E}^{L, 0-}=0 .
\end{aligned}
$$




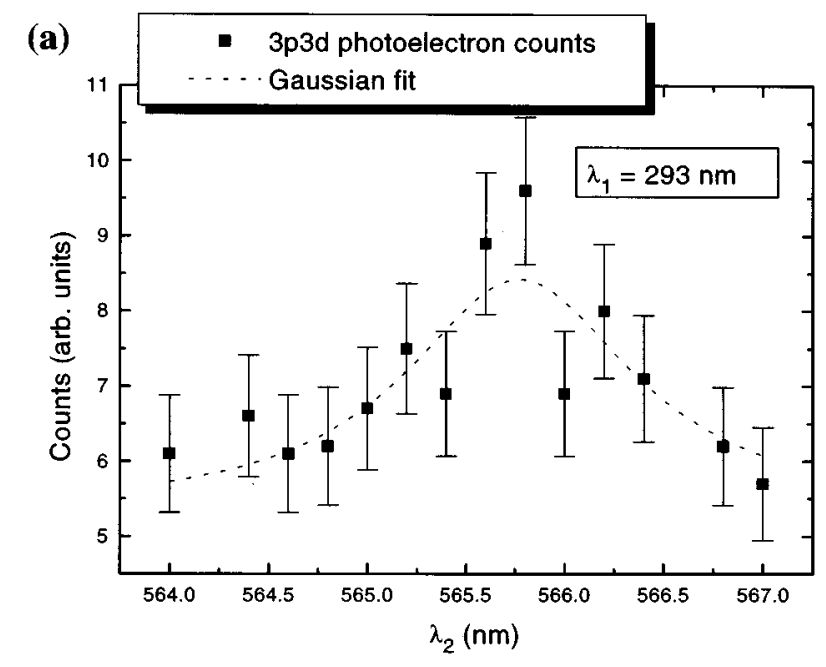

(b)

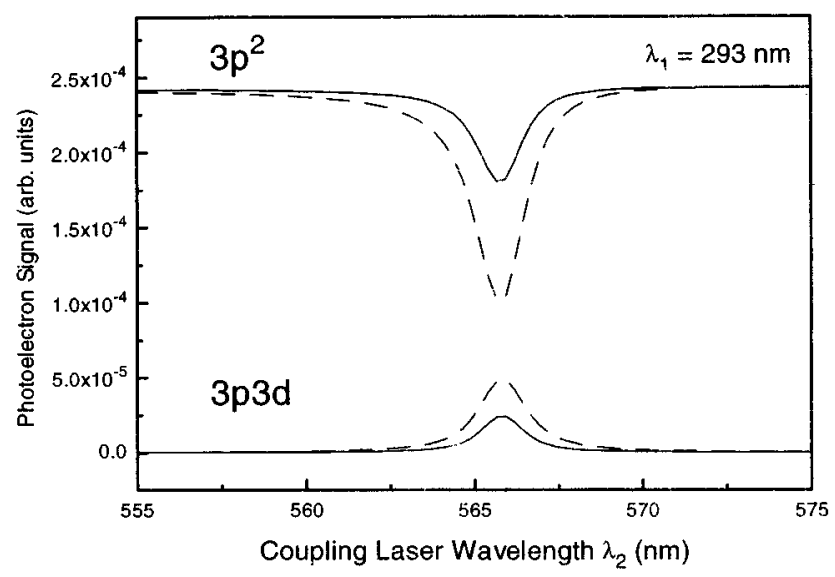

FIG. 7. (a) Energy-selected photoelectron spectrum relating to decay from or around the $3 p 3 d$ AIS $\left(E_{e} \approx 3 \mathrm{eV}\right)$. The squares are the actual data points obtained by a pulse counting method, the error bars are the statistical error of the measurement, and the dotted line is a Gaussian fit to the data, approximating the shape of a resonance. (b) Theoretical calculation of a photoelectron spectrum in the regions of the $3 p^{2}$ and the $3 p 3 d$ AIS with $\lambda_{1}=293 \mathrm{~nm}$. The dashed line indicates full spatial overlap, the full line indicates partial overlap $\left(\kappa_{1} / \kappa_{2}=0.4\right)$.

We see in this expression that the effect of resonant states different from $\phi_{r}^{L}$ is taken into account through the second term of $\mathscr{H}_{v}^{\prime}$. On the other hand, the shift in the energy position of $\phi_{r}^{L}$ is written

$$
\Delta_{r}=\mathscr{P} \oint \frac{\left|\left\langle\phi_{n}^{L}\left|Q \mathscr{H}_{v} P\right| P \psi_{E^{\prime}}^{L, 0-}\right\rangle\right|^{2}}{\left(E-E^{\prime}\right)} d E^{\prime} .
$$

We now consider the two-photon transition from the ground state, $\psi_{g}$, to the energy region close to autoionizing state $\phi_{r}^{L}$. The Fano profile parameter $q(E)$ is given by $([11,20])$

$$
q(E)=\frac{\Omega_{1}(E)}{\pi\left\langle\psi_{g}|T| \psi_{E}^{L, 0-}\right\rangle\left\langle P \psi_{E}^{L, 0-}\left|P \mathscr{H}_{v} Q\right| \phi_{r}^{L}\right\rangle},
$$

where $\psi_{E}^{L, 0-}$ is a nonresonant continuum with a component in $Q$ subspace due to the existence of neighboring resonances:

$$
\psi_{E}^{L, 0-}=P \psi_{E}^{L, 0-}+\oint_{n \neq r} \frac{\left\langle\phi_{n}^{L}\left|Q \mathscr{H}_{v} P\right| P \psi_{E}^{L, 0-}\right\rangle}{\left(E-\mathscr{E}_{n}\right)} \phi_{n}^{L}
$$

and $T$ is the two-photon transition operator:

$$
T=I_{1}(r, t)\left(\lim _{\eta \rightarrow 0^{+}} \oint d E_{i} \frac{\vec{D} \cdot \vec{e}\left|\psi_{i}\right\rangle\left\langle\psi_{i}\right| \vec{D} \cdot \vec{e}}{\left(E+\hbar \omega_{1}-E_{i}-i \eta\right)}\right)
$$

Here, $I_{1}(r, t)$ is the spatial and temporal intensity envelope of laser 1 [see Eq. (21) below], $\left\{\psi_{i}\right\}$ is the sets of all possible intermediate states, $\hbar \omega_{1}$ is the photon energy, $\vec{D}$ is either $\vec{r}$ (length) or $\vec{\nabla} / \hbar \omega_{1}$ (velocity), depending on the chosen representation, and $\vec{e}$ is the polarization vector; it must be remembered that linearly polarized radiation is considered in this work. In the definition of $q(E)$, Eq. $(13), \Omega_{1}(E)$ includes two-photon dipolar couplings between the ground state $\psi_{g}$ and both $\phi_{r}^{L}$ and the set of nonresonant continuum states $\psi_{E}^{L, 0-}$ with energy different from $\mathscr{E}_{r}$ :

$$
\begin{aligned}
\Omega_{1}(E)= & \left\langle\psi_{g}|T| \phi_{r}^{L}\right\rangle \\
& +\mathscr{P} \oint \frac{\left\langle\psi_{g}|T| \psi_{E^{\prime}}^{L, 0-}\right\rangle\left\langle P \psi_{E^{\prime}}^{L, 0-}\left|P \mathscr{H}_{v} Q\right| \phi_{r}^{L}\right\rangle}{\left(E-E^{\prime}\right)} d E^{\prime} .
\end{aligned}
$$

In the density-matrix equations, there also appears $\gamma_{3 s^{2}}(E)$, the ionization width of ground state $\psi_{g}$ to nonresonant continua of symmetries ${ }^{1} S^{e}$ and ${ }^{1} D^{e}$ :

$$
\gamma_{3 s^{2}}(E)=2 \pi\left|\left\langle\psi_{g}|T| \psi_{E}^{S, 0-}\right\rangle\right|^{2}+2 \pi\left|\left\langle\psi_{g}|T| \psi_{E}^{D, 0-}\right\rangle\right|^{2} .
$$

Note that, in the energy region close to $3 p^{2}\left({ }^{1} S^{e}\right)$, the wave function $\psi_{E}^{D, 0-}$ is associated to the resonance-free continuum $3 s k d$ and is directly calculated as an eigenfunction of $\mathscr{H}_{v}$. Finally, the dipolar coupling between the discrete part of resonances $3 p^{2}\left({ }^{1} S^{e}\right)$ and $3 p 3 d\left({ }^{1} P^{o}\right)$ is written

$$
\Omega_{2}=\sqrt{I_{2}(r, t)}\left\langle\phi_{3 p^{2}}^{S}|\vec{D} \cdot \vec{e}| \phi_{3 p 3 d}^{P}\right\rangle,
$$

where $I_{2}(r, t)$ is the spatial and temporal intensity envelope of laser 2. In the above equations, we see that $\Omega_{1}(E)$ is proportional to $I_{1}(r, t), \gamma_{3 s^{2}}(E)$ to $I_{1}^{2}(r, t)$, and $\Omega_{2}$ to $I_{2}^{1 / 2}(r, t)$.

\section{B. Results}

The calculation procedure is similar to the one we have employed in previous works with different two-electron Hamiltonians; the details can be found, for example, in [20]. In a first step, we obtain one-electron orbitals by diagonaliz- 
TABLE I. Atomic parameters of the levels involved in the process. All energies are referred to the double ionization threshold $\mathrm{Mg}^{2+} \cdot \gamma_{3 s^{2}} \equiv \gamma_{3 s^{2}}\left(E=E_{3 p^{2}}\right), \Omega_{1} \equiv \Omega_{1}\left(E=E_{3 p^{2}}\right), q \equiv q\left(E=E_{3 p^{2}}\right)$, and $\Omega_{2}$ have been calculated in the length representation.

\begin{tabular}{lcc}
\hline \hline$E_{3 s^{2}}=-0.8319$ a.u. & $\gamma_{3 s^{2}} / I_{1}^{2}(r, t)=1.45 \times 10^{8}$ a.u. & $q=27.7$ a.u. \\
$E_{3 p^{2}}=-0.5199$ a.u. & $\Gamma_{3 p^{2}}=0.00171$ a.u. & $\Omega_{1} / I_{1}(r, t)=818.4$ a.u. \\
$E_{3 p 3 d}=-0.4418$ a.u. & $\Gamma_{3 p 3 d}=0.000102$ a.u. & $\Omega_{2} / \sqrt{I_{2}(r, t)}=2.224$ a.u. \\
\hline \hline
\end{tabular}

ing $h_{v}\left(\vec{r}_{i}\right)+V_{1}\left(\vec{r}_{i}\right)$ in a basis set of $B$-spline functions [21] that are orthogonalized to the Hartree-Fock core wave function. We have used $190 \mathrm{~B}$-splines per angular momentum defined in a box of 75 a.u.; the $B$-spline order is 10 , and the knot sequence linear. For the core dipole polarizability we have taken $\alpha=0.491$ a.u [22].

In the next step, these orbitals are used to build configurations adapted to the symmetry, and two-electron wave functions are evaluated by performing appropriate configuration interaction $(\mathrm{CI})$ calculations. To obtain the ground state $\psi_{g}$, the set of intermediate states $\left\{\psi_{i}\right\}$, and the set of resonant states $\left\{\phi_{n}^{L}\right\}$ we have included into the basis sets series $\left(3 \ln l^{\prime}\right),\left(4 \ln l^{\prime}\right),\left(5 \ln l^{\prime}\right),\left(6 \ln l^{\prime}\right)$, and $\left(7 \ln l^{\prime}\right)$. In each diagonalization we have around 500 configurations; obviously, series $(3 s n l)$ are excluded to determine $\left\{\phi_{n}^{L}\right\}$. On the other hand, discretized wave functions $\left\{\tilde{\chi}_{E_{i}}^{L, 0-}\right\}$ are calculated in each channel $3 s k l$ by solving Eq. (11) in a basis set of 80 configurations $(3 s n l)$. These solutions approximate continuum states $\left\{P \psi_{E}^{L, 0-}\right\}$ within the box after renormalization:

$$
P \psi_{E}^{L, 0-} \simeq \rho^{1 / 2}\left(E_{i}\right) \tilde{\chi}_{E_{i}}^{L, 0-},
$$

where $E \simeq E_{i}$, and $\rho\left(E_{i}\right)$ is a density of states given by $\rho\left(E_{i}\right)=2 /\left|E_{i-1}-E_{i+1}\right|$. It must be noticed that, as configurations are built with orbitals that are eigenfunctions of $h_{v}\left(\vec{r}_{i}\right)+V_{1}\left(\vec{r}_{i}\right)$, the only terms coupling wave functions of $Q$ and $P$ subspaces are $1 / r_{12}+V_{\text {diel }}\left(\vec{r}_{1}, \vec{r}_{2}\right)$.

The parameters we have obtained are reported in Table I, and compared with other previous theoretical and experimental results, when possible, in Table II. We see that the overall agreement is satisfactory, although slight differences are found. The theoretical studies presented in $[13,16]$ are based on a frozen-core approach with the same polarization potential we have employed here (core polarization was ne- glected in [14]). In both works, energies and widths were extracted from a phase-shift analysis. Discrepancies among their results and ours can be explained by different degrees of electronic correlation introduced in the valence shell. On the other hand, the experimental findings shown in Table II were obtained by studying two-photon ionization processes $[9,23]$, and double excitation in $\mathrm{Mg}-e^{-}$collisions [24]. We note that the $3 p^{2}$ state was first observed by Bradley et al. [25] through sequential excitation via the $3 s 3 p\left({ }^{1} P^{o}\right)$ bound state.

Single-color two-photon ionization of $\mathrm{Mg}$ from its ground state in the vicinity of autoionizing state $3 p^{2}\left({ }^{1} S^{e}\right)$ is near resonant with single-photon absorption to bound state $3 s 3 p\left({ }^{1} P^{o}\right)$. As a consequence, the structure in the ionization rate due to autoionizing state $3 p^{2}\left({ }^{1} S^{e}\right)$ is embedded in a huge peak, and the background associated to the Fano profile varies rapidly with energy (see Fig. 8). This energy dependence also appears in the parameters $\gamma_{3 s^{2}}(E), \Omega_{1}(E)$, and $q(E)$; they are given at the resonance energy in Table I. All of them have a smooth, though non-negligible, dependence with energy, as is illustrated in Fig. 9 for $\gamma_{3 s^{2}}(E)$ and $\Omega_{1}(E)$. Moreover, due to the high sensitivity of the values of these parameters with the relative positions of states $3 s^{2}\left({ }^{1} S^{e}\right), 3 s 3 p\left({ }^{1} P^{o}\right)$, and $3 p^{2}\left({ }^{1} S^{e}\right)$, final results may be affected by small errors in the calculated energies. Because of that, we have shifted states $3 s^{2}\left({ }^{1} S^{e}\right), 3 p^{2}\left({ }^{1} S^{e}\right)$, and $3 p 3 d\left({ }^{1} P^{o}\right)$ making $E_{3 s 3 p}-E_{3 s^{2}}, \quad E_{3 p^{2}}-E_{3 s^{2}}, \quad$ and $E_{3 p 3 d}-E_{3 p^{2}}$ equal to the experimental results: $E_{3 s 3 p}-E_{3 s^{2}}=4.346 \mathrm{eV}$, and $E_{3 p^{2}}-E_{3 s^{2}}=8.4646 \mathrm{eV}$ and $E_{3 p 3 d}-E_{3 p^{2}}=2.191 \mathrm{eV}$ (see [23], and references in paper I). With these values, two-photon ionization from the ground state to $3 p^{2}\left({ }^{1} S^{e}\right)$ is exactly resonant at $\lambda_{1}=292.95 \mathrm{~nm}$, while the single-photon transition between autoionizing states $3 p^{2}\left({ }^{1} S^{e}\right)$ and $3 p 3 d\left({ }^{1} P^{o}\right)$ is resonant at $\lambda_{2}=565.8$ nm.

TABLE II. Comparison of the present results and those obtained in some previous experimental and theoretical works. Energies are now referred to the ground state of Mg. (a) Moccia and Spizzo [14,16], (b) Chang [13], (c) Shao, Fotakis, and Charalambidis [9], (d) Bonano, Clark, and Lucatorto [23], and (e) Rassi et al. [24].

\begin{tabular}{lccccccc}
\hline \hline & \multicolumn{3}{c}{ Theory } & & \multicolumn{3}{c}{ Experiment } \\
\cline { 2 - 3 } \cline { 6 - 8 } & Present & (a) & (b) & $($ c) & (d) & (e) \\
\hline$E_{3 p^{2}}$ (a.u.) & 0.3120 & $0.3113^{\mathrm{a}}$ & 0.3104 & & 0.3111 & 0.3111 & 0.3105 \\
$\Gamma_{3 p^{2}}$ (a.u.) & 0.00171 & $0.00172^{\mathrm{a}}$ & 0.00123 & & 0.00128 & 0.00127 & \\
$E_{3 p 3 d}$ (a.u.) & 0.3901 & $0.3984^{\mathrm{b}}$ & & & & & 0.3910 \\
$\Gamma_{3 p 3 d}$ (a.u.) & 0.000102 & $0.000092^{\mathrm{b}}$ & & & & \\
\hline \hline
\end{tabular}

${ }^{\mathrm{a}}$ Moccia and Spizzo [16].

${ }^{\mathrm{b}}$ Moccia and Spizzo [14]. 


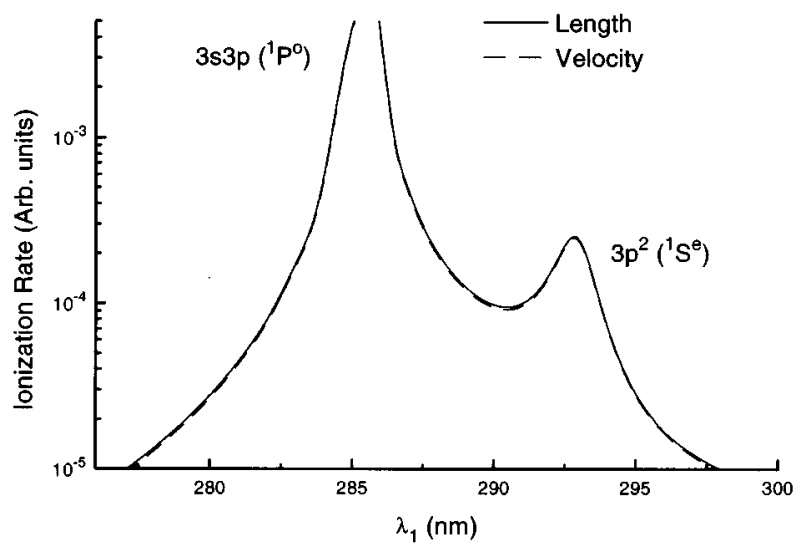

FIG. 8. Two-photon ionization rate in the vicinity of autoionizing state $3 p^{2}\left({ }^{1} S^{e}\right)$. The structure due to one-photon resonance with the bound state $3 s 3 p\left({ }^{1} P^{o}\right)$ is also shown. Laser 2 is off.

\section{DYNAMICS, DENSITY-MATRIX EQUATIONS}

\section{A. Density-matrix equations}

We recall below the density-matrix equations governing the time evolution of the populations. The indices 1,2 , and 3 are associated to the states $3 s^{2}\left({ }^{1} S^{e}\right), 3 p^{2}\left({ }^{1} S^{e}\right)$, and $3 p 3 d\left({ }^{1} P^{o}\right)$, respectively;

$$
\begin{aligned}
& \frac{d \rho_{11}}{d t}=-\gamma_{3 s^{2}}\left(E_{f}\right) \rho_{11}+2 \operatorname{Im}\left[\Omega_{1}\left(E_{f}\right)\left(1-\frac{i}{q\left(E_{f}\right)}\right) \rho_{21}\right], \\
& \frac{d \rho_{22}}{d t}=-\Gamma_{3 p^{2}} \rho_{22}-2 \operatorname{Im}\left[\Omega_{1}\left(E_{f}\right)\left(1+\frac{i}{q\left(E_{f}\right)}\right) \rho_{21}\right] \\
& +2 \operatorname{Im}\left(\Omega_{2} \rho_{32}\right), \\
& \frac{d \rho_{33}}{d t}=-\Gamma_{3 p 3 d} \rho_{33}-2 \operatorname{Im}\left(\Omega_{2} \rho_{32}\right), \\
& \frac{d \rho_{21}}{d t}=-\left[-i \delta_{1}+\frac{\left(\gamma_{3 s^{2}}+\Gamma_{3 p^{2}}\right)}{2}\right] \rho_{21}-i \Omega_{1}\left(E_{f}\right) \\
& \times\left[\left(1-\frac{i}{q\left(E_{f}\right)}\right) \rho_{11}-\left(1+\frac{i}{q\left(E_{f}\right)}\right) \rho_{22}\right]-i \Omega_{2} \rho_{31}, \\
& \frac{d \rho_{31}}{d t}=-\left[-i\left(\delta_{1}+\delta_{2}\right)+\frac{\left(\gamma_{3 s^{2}}\left(E_{f}\right)+\Gamma_{3 p 3 d}\right)}{2}\right] \rho_{31}+i \Omega_{1}\left(E_{f}\right) \\
& \times\left(1+\frac{i}{q\left(E_{f}\right)}\right) \rho_{32}-i \Omega_{2} \rho_{21}, \\
& \frac{d \rho_{32}}{d t}=-\left[-i \delta_{2}+\frac{\left(\Gamma_{3 p^{2}}+\Gamma_{3 p 3 d}\right)}{2}\right] \rho_{32}+i \Omega_{1}\left(E_{f}\right) \\
& \times\left(1+\frac{i}{q\left(E_{f}\right)}\right) \rho_{31}-i \Omega_{2}\left(\rho_{22}-\rho_{33}\right),
\end{aligned}
$$

with $\delta_{1}=2 \hbar \omega_{1}-\left(E_{3 p^{2}}-E_{3 s^{2}}\right) \quad$ and $\quad \delta_{2}=\hbar \omega_{2}$ $-\left(E_{3 p 3 d}-E_{3 p^{2}}\right) . E_{f}=E_{3 s^{2}}+2 \hbar \omega_{1}$. Note that the energy dependence of the parameters $\gamma_{3 s^{2}}, \Omega_{1}$, and $q$ is explicitly

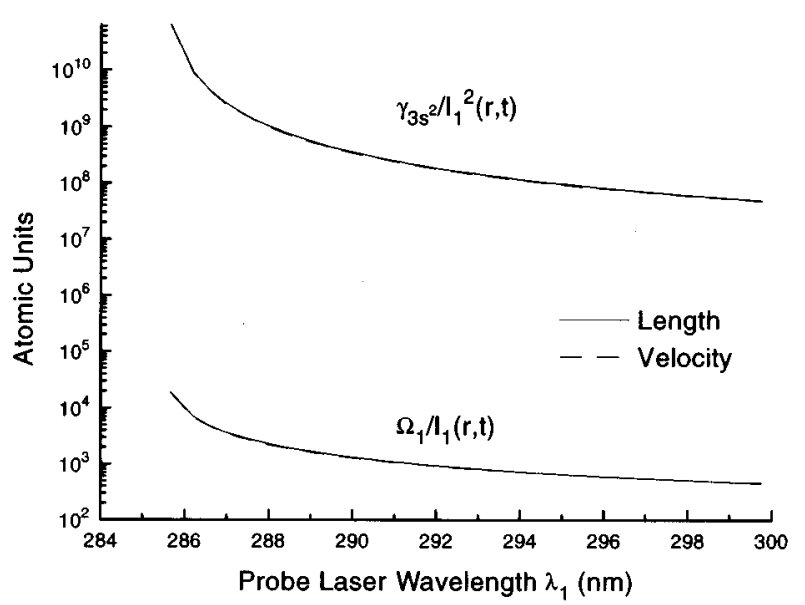

FIG. 9. Energy dependence of some atomic parameters. See definitions in text [Eqs. (16) and (17)].

taken into account in the calculations. $\Gamma_{3 p^{2}, 3 p 3 d}$ represents the $\left(3 p^{2}, 3 p 3 d\right)$ AIS autoionization widths.

\section{B. Treatment of the spatial and temporal dependence of the fields}

We assume a cylindrical symmetry $(r, z)$ for the space macrodependence of the intensities for both lasers, $z$ being the propagation direction. Thus, the quantities involved in Eqs. (20) being dependent on $r$, the populations $\rho_{i i}(r, t=\infty)$ must be integrated over $r$. The temporal dependence is assumed to have a $\cosh ^{-1}$ form; we take a Gaussian radial dependence (in space). The intensity envelope is written

$$
I_{i}(r, t)=\frac{e^{-\left(4 \ln 2 / \kappa_{i}^{2}\right) r^{2}}}{\left(\cosh 1.76 t / \tau_{i}\right)^{2}} I_{i}^{\max }
$$

Here, $i$ labels the laser ( 1 or 2 ). $\tau_{i}$ and $\kappa_{i}$ are the full width at half maximum (FWHM) values for temporal and spatial intensity envelopes, respectively. The ionization amount $P^{I}$ is given by

$$
P^{I}=\int_{0}^{\infty}\left[1-\rho_{11}(r, t=\infty)\right] r d r .
$$

Note that, since we are not interested in absolute measurements, we have neglected proportionality factors in the above formula. The pulse duration $\left(\tau_{i}\right)$ is taken as a picosecond for both lasers, as we have checked that, under the experimental conditions, the total ionization amount is proportional to the interaction time. This is because the steady regime has been reached, and, as long as no saturation effects occur, the ionization can be expressed as a rate. The quantity of electrons emitted in the $3 p 3 d$ region ( $3 s k p$ continuum) is given by the formula

$$
P_{3 p 3 d}^{I}(r)=\int_{-\infty}^{\infty} \rho_{33}(r, t) \Gamma_{3 p 3 d} d t .
$$



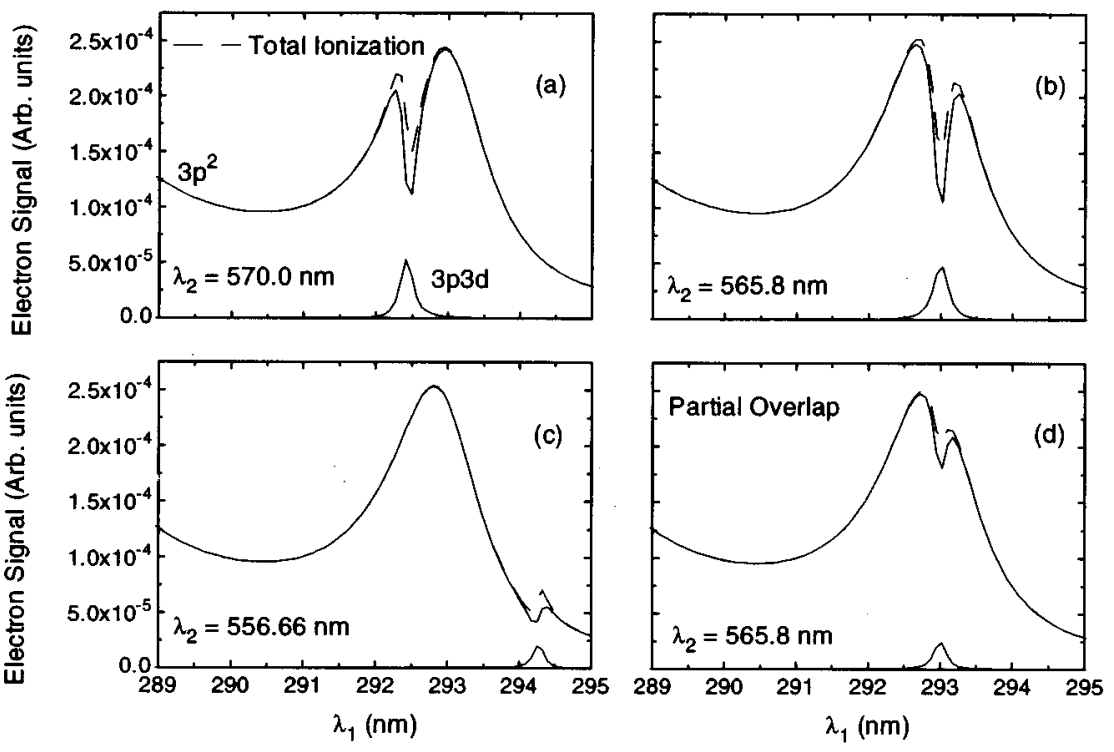

FIG. 10. (a)-(c) Partial and total ionization spectra calculated for different values of $\lambda_{2}$ with full spatial overlap. (d) Same as (b) but with partial overlap $\left(\kappa_{2} / \kappa_{1}=0.4\right)$.
The total quantity $P_{3 p 3 d}^{I}$ is obtained after integration over the space. The rest of the emitted electrons go in the $3 p^{2}$ region, then

$$
P_{3 p^{2}}^{I}=P^{I}-P_{3 p 3 d}^{I}
$$

\section{Results}

All calculations have been performed assuming a linear polarization of the laser fields, $I_{1}^{\max }=10^{8} \mathrm{~W} / \mathrm{cm}^{2}$ and $I_{2}^{\max }=0.25 \times 10^{10} \mathrm{~W} / \mathrm{cm}^{2}$. In Fig. 8, we show the total ionization signal when laser 2 is off. We observe a divergent structure due to the presence of the $3 s 3 p\left({ }^{1} P^{o}\right)$ bound state (see Sec. IV B) and the Fano profile corresponding to the excitation of the $3 p^{2}\left({ }^{1} S^{e}\right)$ resonance. Calculations have been performed in velocity and length gauges; we see in Fig. 8 that there is a perfect agreement.

In our calculations, the $3 p 3 d$ discrete level is coupled to the continuum only through autoionization. Indeed, dipole couplings between $3 p 3 d$ and $3 s k l$ states are expected to be small since their dominant configurations do not share common orbitals. The same remark holds for the dipole coupling between the discrete $3 p^{2}$ state and the upper $3 s k p$ continuum (in the $3 p 3 d$ region). Thus, on the basis of these considerations, the ionization in the $3 p 3 d$ region only results from the autoionization of the upper AIS; this justifies the formula (23) for the calculation of ionization in that region. We have also numerically checked that the photoionization of the upper AIS to the $3 p k l^{\prime}$ continua is negligible.

Figures 10(a)-10(d) and 4(b) report on two-color effects; (i) the wavelength of laser 2 is fixed and $\lambda_{1}$, the probe laser wavelength, is varied (Fig. 10), (ii) $\lambda_{1}$ is fixed and the wavelength of laser 2 is scanned [Fig. 4(b)]. Except in the case of Fig. 10(d), which is to be compared to Fig. 10(b), we have taken $\kappa_{2} / \kappa_{1}=1$ [see Eq. (21)]; in our model, this corresponds to maximum spatial overlap between lasers 1 and 2 . In all figures we have reported total ionization, and in addition we have also shown the contribution of $3 p^{2}$ and $3 p 3 d$ resonance region in Fig. 10. We observe that electrons are mainly emitted in the $3 p^{2}\left({ }^{1} S^{e}\right)$ region. As a consequence,
$3 p^{2}\left({ }^{1} S^{e}\right)$ electron spectra are very similar to the total ionization spectra (ion yield) reported in paper I. As expected, the structures in the ionization profiles are less pronounced when there is no full overlap [see Figs. 10(b) and 10(d)].

Figure 7 (b) shows the electron spectra in the regions of $3 p^{2}$ and $3 p 3 d$ resonances for $\lambda_{1}$ fixed at $293 \mathrm{~nm}$. Different spatial overlaps are shown in the figure, and in all cases we see that the electron emission from $3 p 3 d\left({ }^{1} P^{o}\right)$ has a peak when the resonance condition is fulfilled $\left(\delta_{1}+\delta_{2}=0\right)$, while the emission from $3 p^{2}\left({ }^{1} S^{e}\right)$ level drops. Therefore, at threephoton resonance, the relative population amount trapped in the upper $3 p 3 d$ state is more important. This confirms the similarity with the $\Lambda$ system evoked in paper I. Nevertheless, one must note that, at three-photon resonance, the total amount of population in $3 p^{2}$ and $3 p 3 d$ AIS discrete parts decreases dramatically, thus the influence of AIS on ionization vanishes. This situation is numerically well illustrated by the simple formula obtained in paper I for the ionization rate:

$$
\begin{aligned}
\frac{d P}{d t} \approx & \gamma_{3 s^{2}}\left(E_{f}\right) \\
& +\Gamma_{3 p^{2}} \frac{\left[\Omega_{1}\left(E_{f}\right)\left(\delta_{1}+\delta_{2}\right)\right]^{2}}{\left[\delta_{1}\left(\delta_{1}+\delta_{2}\right)-\Omega_{2}^{2}\right]^{2}+\left[\Gamma_{3 p^{2}}\left(\delta_{1}+\delta_{2}\right) / 2\right]^{2}} .
\end{aligned}
$$

Note that an exponent 2 is missing in paper I [Eq. (2), above $\Omega_{1}$ ]. This formula has been obtained for square pulses and weak field conditions.

\section{DISCUSSION}

A comparison of the results given in the previous sections shows that theory and experiment are in very good agreement in all the cases examined, within the limits of experimental uncertainties such as intensity and overlap. This allows for a thorough interpretation of the effects observed in terms of phenomena known and demonstrated in other systems, i.e., ac Stark splitting and population trapping. Effec- 
tively, ac Stark splitting between two autoionizing states is the dominant mechanism behind the behavior observed and, although similar effects have long been known to occur through the strong coupling between two bound states, it is the first time that the phenomenon is experimentally demonstrated in a system in the continuum. Thus, in the case where $\delta_{1}=0$, the dip in Figs. 3 and 7(b) is the transparency produced because of the splitting of the $3 p^{2}$ state through the strong resonant coupling to the $3 p 3 d$ by the intense laser, which is demonstrated as a reduction in ionization. Although the $3 p^{2}$ AIS is broad as compared to the Rabi frequency the splitting can nevertheless be observed; the $3 p 3 d$ state must also be split, but this effect cannot be probed with the present setup and requires a direct probing of the higher state through the ground state. Theoretical treatment of ac Stark splitting of autoionizing states [7] indicates that the splitting can be asymmetric in the case of exact resonance, although it is not possible to establish this here due to the uncertainty in our wavelength determination of the center of the state.

The electron spectroscopy provides information on the relative contribution of discrete $3 p^{2}$ and $3 p 3 d$ levels to the ionization process. Note that, in a steady regime and square pulse condition, the populations of these levels are constant in time. The calculation of the relative population amount in levels $3 p^{2}$ and $3 p 3 d$ shows that, except for the three-photon resonant case $\left(\delta_{1}+\delta_{2}=0\right)$, the $3 p^{2}$ discrete state is much more populated than the upper AIS. At three-photon resonance, the upper AIS may be more populated than the lower one; nevertheless the total population amount of both discrete levels drops in this case and the AIS contribution to ionization is greatly reduced. This is confirmed by the experimental observations which show that all structures in the ionization spectra are found in the $3 p^{2}$ region. For the sake of clarity in explaining the ionization quasisuppression at three-photon resonance, it is worth noting that it is not related to population trapping in autoionizing states. On the other hand, due to destructive interference effects at resonance, the total amount of population present in the two deexcited states dramatically decreases in this case. In view of a more general prospect, complete stabilization could be obtained in the absence of noninterfering ionization background, for example, by using an appropriate combination of AIS and laser polarizations. All effects will, of course, be limited by intensity averaging due to the pulse shape and imperfect beam overlap.

As the coupling laser becomes detuned from resonance [see Figs. 4(a) and 4(b)], the splitting becomes asymmetric as expected, displaying a dip at the position of the coupling and a peak where the split component is expected, with the relative position of the dip and peak (and therefore the asymmetry) changing as the coupling switches to the other side of the $3 p^{2}$ resonance. This becomes more clear as the detuning increases beyond the width of the $3 p^{2}$ state, where the split component becomes apparent in both the total ionization and energy-selected photoelectron spectra (see Figs. 5 and 6). Although part of the ionization signal in these cases arises from the three-photon excitation of the $3 p 3 d$ state, as shown in the theoretical spectra (see Fig. 10), the fact that experimentally the peak is observed in the $3 p^{2}$ photoelectron region, and the signal from the $3 p 3 d$ is almost negligible indicates that ac Stark splitting significantly contributes to the appearance of the small peak. We have noted in Sec. V C that we have neglected the ionization resulting from the $3 p 3 d$ $3 s k l$ dipole coupling in the $3 p^{2}$ region. Nevertheless such approximations may not be valid when laser 2 is tuned far from $3 p^{2}-3 p 3 d$ resonance. In the latter case autoionization from the $3 p^{2}$ state may have a minor contribution compared to other ionization sources, such as the photoionization channel described above. Actually, the nonresonant case is very closely related to the scheme of LICS. In the past, LICS in a smooth continuum have been investigated in $\Lambda$ systems involving a bound state which is electromagnetically embedded in the continuum. In fact, if the $3 p^{2}$ were not present in the scheme under investigation this would be exactly a ladder LICS scheme, in which the bound state is replaced by an AIS. This AIS is then embedded by $\hbar \omega_{2}$ at a lower position in the continuum. In LICS in a smooth continuum that involves either a $\Lambda$ or a ladder system, the virtual state of the process (Raman or direct) coupling the two discrete states can be considered as being made from the ac Stark split components of all the parity and angular-momentum allowed discrete states. In the presence of the $3 p^{2}$, in the nonresonant case, it is this state that might have the dominant contribution to the virtual state of the three-photon coupling, and the overall scheme can be understood as an "above-threshold" LICS scheme.

By this means, the investigated phenomenon can be considered to be very relevant to the above-threshold ionization (ATI) process occurring at higher laser intensities and short pulse durations. It is well known that in such experiments bound states can be ac Stark shifted into resonance with $n$ photons of the field. These transient resonances (also called Freeman resonances [26]) are known to induce structure in the above-threshold processes, e.g., in the ATI photoelectron peaks, due to the resonantly enhanced ionization cross section. Our present results demonstrate that an "abovethreshold" LICS effect could lead to the observation of structure in high-intensity above-threshold processes due to the ac Stark shifting of AIS into resonance. This induced structure is expected to be present in all the ATI peaks above and below the shifted AIS.

In conclusion, the effects observed in our study and their direct relevance to above-threshold processes make the results presented very significant in the field of modification and control of the properties of atomic continua, with a view to understanding the continuum interactions with an electromagnetic field and to making use of these modifications in an effectively unlimited energy range above the threshold, within the capabilities of available laser sources.

\section{ACKNOWLEDGMENTS}

The experimental work presented was conducted in the laboratories of the Ultraviolet Laser Facility operating at FORTH-IESL (Human Capital and Mobility, Access to Large Scale Facilities EU programme, Contract No. CHGE-CT92007). Part of this work has been conducted under Human Capital and Mobility Contract No. CHRX-CT94-0695. One of us (I.S.) wishes to acknowledge the "Ministerio de Educación y Ciencia" (Spain) for funding through program MEC/MRE. 
[1] Y. L. Shao, D. Charalambidis, C. Fotakis, Jian Zhang, and P. Lambropoulos, Phys. Rev. Lett. 67, 3669 (1991); S. Cavalieri, F. S. Pavone, and C. Matera, ibid. 67, 3673 (1991); O. Faucher, Y. L. Shao, D. Charalambidis, and C. Fotakis, Phys. Rev. A 50, 641 (1994), and references therein.

[2] K. -J. Boller, A. Imamoglu, and S. E. Harris, Phys. Rev. Lett. 66, 2593 (1991).

[3] A. Aspect, E. Arimondo, R. Kaiser, N. Vansteenkiste, and C. Cohen-Tannoudji, Phys. Rev. Lett. 61, 826 (1988), and references therein.

[4] J. R. Kuklinski, U. Gaubatz, F. T. Hioe, and K. Bergmann, Phys. Rev. A 40, 6741 (1989).

[5] H. G. Muller and M. P. de Boer, in Proceedings of the 6th International Conference, Multiphoton Processes 1993, edited by D. K. Evans and S. L. Chin (World Scientific, Singapore, 1994), and references therein.

[6] N. E. Karapanagioti, O. Faucher, Y. L. Shao, D. Charalambidis, H. Bachau, and E. Cormier, Phys. Rev. Lett. 74, 2431 (1995).

[7] P. Lambropoulos and P. Zoller, Phys. Rev. A 24, 379 (1981).

[8] H. Bachau, P. Lambropoulos, and Robin Shakeshaft, Phys. Rev. A 34, 4785 (1986).

[9] Y. L. Shao, C. Fotakis, and C. Charalambidis, Phys. Rev. A 48, 3636 (1993).
[10] V. Lange, U. Eichmann, and W. Sandner, J. Phys. B 22, L245 (1989).

[11] U. Fano, Phys. Rev. 124, 1866 (1961).

[12] J. Gea-Banacloche, Y. Li, S. Jin, and M. Xiao, Phys. Rev. A 51, 576 (1995).

[13] T. N. Chang, Phys. Rev. A 36, 5468 (1987).

[14] R. Moccia and P. Spizzo, J. Phys. B 21, 1121 (1988).

[15] R. Moccia and P. Spizzo, J. Phys. B 21, 1145 (1988).

[16] R. Moccia and P. Spizzo, Phys. Rev. A 39, 3855 (1989).

[17] D. W. Norcross and M. J. Seaton, J. Phys. B 9, 2983 (1976).

[18] H. Feshbach, Ann. Phys. (N.Y.) 19, 287 (1962).

[19] Y. Hahn, T. F. O’Malley, and L. Spruch, Phys. Rev. 128, 932 (1962).

[20] I. Sánchez, H. Bachau, and E. Cormier, J. Phys. B 28, 2367 (1995).

[21] C. de Boor, A Practical Guide to Splines (Springer, New York, 1978).

[22] C. Mendoza, J. Phys. B 14, 397 (1981).

[23] R. E. Bonano, C. W. Clark, and T. B. Lucatorto, Phys. Rev. A 34, 2082 (1986).

[24] D. Rassi, V. Pejcev, T. W. Ottley, and K. J. Ross, J. Phys. B 10, 2913 (1977).

[25] D. J. Bradley, C. H. Dugan, P. Ewart, and A. F. Purdie, Phys. Rev. A 13, 1416 (1976).

[26] R. R. Freeman and P. H. Bucksbaum, J. Phys. B 24, 325 (1991), and references therein. 